\title{
HIGHER ORDER ESTIMATES IN FURTHER DIMENSIONS FOR THE SOLUTIONS OF NAVIER-STOKES EQUATIONS
}

\author{
MICHAEL WIEGNER \\ Department of Mathematics I, RWTH Aachen \\ D-52056 Aachen, Germany \\ E-mail:wiegner@math1.rwth-aachen.de
}

The aim of this note is to continue the study of the decay with respect to $t \rightarrow \infty$ of higher order norms of strong solutions of the Navier-Stokes equations in the whole space $\mathbb{R}^{n}$, which was first addressed in a paper of M. E. Schonbek and M. Wiegner [3].

In general (for $n \geq 3$ ) a smallness assumption is needed for ensuring existence of such a solution. T. Kato [2] (see also [6]) showed that

$$
\begin{aligned}
u_{t}-\triangle u+(u \nabla) u+\nabla p & =0 \\
\operatorname{div} u & =0 \quad \text { on }(0, \infty) \times \mathbb{R}^{n} \\
u(0) & =a
\end{aligned}
$$

has a global solution $u$, smooth for $t>0$, provided $a \in L_{n, \sigma}$ with $\|a\|_{n}$ small enough. Here $L_{n, \sigma}$ denotes the divergence free part of $L_{n}$. If moreover additionally $a \in L_{2}$, the solution has also finite energy for all times and coincides with the Leray-Hopf solution, for which the energy $\|u(t)\|_{2}^{2}$ tends to zero (see e.g. [4]). Note that for $n=3,4$, all Leray-Hopf solutions with generalized energy inequality fulfil the necessary smallness assumption after some time $T_{0}$; see the discussion in [3] or [6].

Suppose now that $\|u(t)\|_{2} \leq c \varphi(t)$ with $\varphi(0) \leq 1$ and

$$
\varphi(t) \searrow 0 \text { for } t \nearrow \infty \text { and } \varphi(\gamma t) \leq c_{\gamma} \varphi(t) \text { for all } t>0, \gamma<1
$$

with $c_{\gamma}$ independent of $t$. This is surely true for algebraic decay rates $\varphi(t)=(1+t)^{-\mu}$ with $\mu>0$, which in turn may be concluded from additional assumptions on the initial value, as e.g. $a \in L_{p}$ with some $p, 1 \leq p<2$, see e.g. Wiegner [4].

From [7] we then know that for $t>0,\|u(t)\|_{\infty} \leq c \phi(t) t^{-n / 4}$ and $\left\|D^{\alpha} u(t)\right\|_{2} \leq$ $c \varphi(t) t^{-1}$ for $|\alpha|=2$.

2000 Mathematics Subject Classification: 35Q30, 76D05.

The paper is in final form and no version of it will be published elsewhere. 
Now the question arises how higher order derivatives behave. Note that even the finiteness of $\left\|D^{\alpha} u(t)\right\|_{2}$ for $|\alpha| \geq 3$ is not a priori clear. In fact, in [3], M. E. Schonbek and the author had to restrict the dimension to $n \leq 5$, in order to show that $\left\|D^{\alpha} u(t)\right\|_{2} \leq$ $c t^{-(|\alpha| / 2+\mu)}$ for all $\alpha$ if $\varphi(t)=(1+t)^{-\mu}$.

We are going to prove the same result also in dimension 6 and 7 and at the same time simplify the proof for $n \leq 5$. It seems likely that also pointwise space-time estimates may be derived as in the paper [1] of Amrouche, Girault, M. E. and T. P. Schonbek, which used the results of [3], hence was also restricted to $n \leq 5$.

THEOREM. Let $n \leq 7, a \in L_{n} \cap L_{2}$ with $\|a\|_{n}$ small enough, so that $u(t)$ is a global smooth solution. Let $\|u(t)\|_{2} \leq c \varphi(t)$ with $\varphi$ fulfilling (1). Then

$$
\left\|D^{\alpha} u(t)\right\|_{2} \leq c \varphi(t) t^{-|\alpha| / 2} \quad \text { for all } \alpha \in \mathbb{N}_{0}^{n} .
$$

For the proof we start with

LEMMA 1. Let

$$
\left\|D^{2} v\right\|_{2}^{2}=\sum_{|\alpha|=2}\left\|D^{\alpha} v\right\|_{2}^{2}
$$

Then

$$
\left\|D^{2}\left(u_{j} u_{k}\right)\right\|_{2}^{2} \leq c\|u\|_{\infty}^{2}\left\|D^{2} u\right\|_{2}^{2}
$$

Proof. As

$$
\int\left(\frac{\partial u_{j}}{\partial x_{\alpha}}\right)^{2}\left(\frac{\partial u_{k}}{\partial x_{\beta}}\right)^{2} d x=-\int u_{j} \frac{\partial^{2} u_{j}}{\partial x_{\alpha}^{2}}\left(\frac{\partial u_{k}}{\partial x_{\beta}}\right)^{2} d x-2 \int u_{j} \frac{\partial u_{j}}{\partial x_{\alpha}} \frac{\partial u_{k}}{\partial x_{\beta}} \frac{\partial^{2} u_{k}}{\partial x_{\alpha} \partial x_{\beta}} d x
$$

we get

$$
\int|\nabla u|^{4} d x \leq c \int|u|\left|D^{2} u\right||\nabla u|^{2} d x
$$

hence

$$
\int|\nabla u|^{4} d x \leq \tilde{c} \int|u|^{2}\left|D^{2} u\right|^{2} d x .
$$

Therefore

$$
\left\|D^{\alpha}\left(u_{j} u_{k}\right)\right\|_{2}^{2} \leq c \int\left|D^{\alpha} u\right|^{2}|u|^{2} d x+c \int\left|\nabla u_{j}\right|^{2}\left|\nabla u_{k}\right|^{2} d x \leq c \int\left|D^{2} u\right|^{2}|u|^{2} d x,
$$

implying the claim.

The next lemma gives a convolution estimate.

Lemma 2. Let $\widehat{v}(\xi)$ denote the Fourier transform. Then for $q>0,1 \leq p \leq 2$,

$$
\left\||\xi|^{q} \widehat{u_{j} \cdot u_{k}}(\xi)\right\|_{r} \leq c\|\widehat{u}\|_{p}\left\|\widehat{u}(\xi)|\xi|^{q}\right\|_{2} \quad \text { if } \quad 1+\frac{1}{r}=\frac{1}{p}+\frac{1}{2} .
$$

Proof. As

$$
\widehat{u_{j} \cdot u_{k}}(\xi)=\widehat{u_{j}} * \widehat{u_{k}},
$$

we have

$$
\left|\widehat{u_{j} \cdot u_{k}}(\xi)\right| \leq \int_{\mathbb{R}^{n}}|\widehat{u}(\mu)||\widehat{u}(\xi-\mu)| d \mu .
$$


Therefore

$$
\begin{gathered}
\left.\left.|| \xi\right|^{q} \widehat{u_{j} u_{k}}(\xi)\left|\leq c \int\right| \widehat{u}(\mu)|| \mu\right|^{q}|\widehat{u}(\xi-\mu)| d \mu+c \int|\widehat{u}(\mu)||\widehat{u}(\xi-\mu)||\xi-\mu|^{q} d \mu \\
\Rightarrow\left\|\left.|| \xi\right|^{q} \widehat{u_{j} u_{k}}(\xi)\right\|_{r} \leq c\|\widehat{u}\|_{p}\left\|\widehat{u}(\xi)|\xi|^{q}\right\|_{2}
\end{gathered}
$$

by the convolution estimate.

Now we are in the position to prove the theorem.

ProOF OF THE THEOREM. With the help of the Fourier transformation the solution may be represented as

$$
\widehat{u_{i}}(t, \xi)=\left(\delta_{i j}-\xi_{i} \xi_{j}|\xi|^{-2}\right)\left(e^{-t|\xi|^{2} / 2} \widehat{u_{j}}(t / 2, \xi)-\sum_{k} \int_{t / 2}^{t} e^{-(t-s)|\xi|^{2}} i \xi_{k} \cdot \widehat{u_{j} u_{k}}(s, \xi) d s\right)
$$

Choose first $p$ with $\max \left\{1, \frac{2 n}{6+n}\right\}<p<\frac{n}{n-1}$ (this causes the dimension restriction). Then

$$
\begin{aligned}
\|\widehat{u}(t)\|_{p} \leq & \left\|e^{-t|\xi|^{2} / 2} \widehat{u}(t / 2)\right\|_{p}+\sum_{j, k} \int_{t / 2}^{t}\left\|e^{-(t-s)|\xi|^{2}} \xi_{k} \widehat{u_{j} u_{k}}(s, \xi)\right\|_{p} d s \\
\leq & c\|\widehat{u}(t / 2)\|_{2}\left\|e^{-t|\xi|^{2} / 2}\right\|_{2 p /(2-p)} \\
& \quad+c \sum_{j, k} \int_{t / 2}^{t}\left\||\xi|^{-1} e^{-(t-s)|\xi|^{2}}\right\|_{2 p /(2-p)}\left\||\xi|^{2} \widehat{u_{j} u_{k}}\right\|_{2} d s \\
\leq & c \varphi(t) t^{-n(2-p) /(4 p)}+c \int_{t / 2}^{t}(t-s)^{-n(2-p) /(4 p)+1 / 2}\|u(s)\|_{\infty}\left\|D^{2} u(s)\right\|_{2} d s \\
\leq & c \varphi(t) t^{-n(2-p) /(4 p)}+c t^{3 / 2-n(2-p) /(4 p)} \varphi^{2}(t) t^{-1-n / 4}
\end{aligned}
$$

if $-\frac{n}{4 p}(2-p)+\frac{1}{2}>-1 \Leftrightarrow p>\frac{2 n}{6+n}$.

Hence $\|\widehat{u}(t)\|_{p} \leq c \varphi(t) t^{-n(2-p) /(4 p)}$.

Now we may estimate after multiplication by $|\xi|^{m}$ :

$$
\begin{aligned}
& \left\||\xi|^{m} \widehat{u}(t)\right\|_{2} \leq\left\||\xi|^{m} e^{-t|\xi|^{2} / 2} \widehat{u}(t / 2)\right\|_{2} \\
& \quad+c \sum_{j, k} \int_{t / 2}^{t}\left\||\xi|^{q} \widehat{u_{j} u_{k}}(\xi)\right\|\left\|_{r}\right\||\xi|^{m+1-q} e^{-(t-s)|\xi|^{2}} \|_{2 r /(r-2)} d s
\end{aligned}
$$

with $r=\frac{2 p}{2-p}>2$ and get

$$
\begin{aligned}
& \left\||\xi|^{m} \widehat{u}(t)\right\|_{2} \leq c t^{-m / 2}\|u(t / 2)\|_{2} \\
& \quad+c \int_{t / 2}^{t}\|\widehat{u}(s)\|_{p}\left\||\xi|^{q} \widehat{u}(s)\right\|_{2}(t-s)^{-(1+\varepsilon) / 2-n(r-2) /(4 r)} d s
\end{aligned}
$$

with $q=m-\varepsilon, \varepsilon>0$.

Suppose now that

$$
\left\||\xi|^{q} \widehat{u}(t)\right\|_{2} \leq c \varphi(t) t^{-q / 2}
$$


Then

$$
\begin{aligned}
& \left\||\xi|^{m} \widehat{u}(t)\right\|_{2} \leq c \varphi(t) t^{-m / 2} \\
& \quad+c \int_{t / 2}^{t} \varphi^{2}(s) s^{-n(2-p) /(4 p)} s^{-q / 2}(t-s)^{-(1+\varepsilon) / 2-n(1-1 / p) / 2} d s
\end{aligned}
$$

as $\frac{r-2}{2 r}=\left(\frac{2 p}{2-p}-2\right) /\left(\frac{4 p}{2-p}\right)=1-\frac{1}{p}$, and therefore

$$
\left\||\xi|^{m} \widehat{u}(t)\right\|_{2} \leq c \varphi(t) t^{-m / 2}+c \varphi^{2}(t) t^{-m / 2} t^{n / 4+1 / 2-n / 2} \leq c \varphi(t) t^{-m / 2},
$$

provided $\frac{1+\varepsilon}{2}+\frac{n}{2}\left(1-\frac{1}{p}\right)<1 \Leftrightarrow \varepsilon+n\left(1-\frac{1}{p}\right)<1$. Such an $\varepsilon>0$ can be chosen if $p<\frac{n}{n-1}$; hence the estimate $(*)$ can be improved by induction for all $q>0$, giving $\left\|D^{k} u(t)\right\|_{2} \leq c_{k} \varphi(t) t^{-k / 2}$ for all $k \in \mathbb{N}_{0}$.

REMARK. By interpolation, for $2 \leq p \leq \infty$ and $j \in \mathbb{N}_{0}$ we have the estimate

$$
\left\|D^{j} u(t)\right\|_{p} \leq c_{j, p} \varphi(t)^{-j / 2-n(1 / 2-1 / p) / 2} .
$$

\section{References}

[1] C. Amrouche, V. Girault, M. E. Schonbek, T. P. Schonbek, Pointwise decay of solutions and of higher derivatives to Navier-Stokes equations, SIAM J. Math. Anal. 31 (2000), 740-753.

[2] T. KATO, Strong $L^{p}$-solutions of the Navier-Stokes equation in $R^{m}$, with applications to weak solutions, Math. Z. 187 (1984), 471-480.

[3] M. E. Schonbek, M. Wiegner, On the decay of higher-order norms of the solutions of Navier-Stokes equations, Proc. Roy. Soc. Edinburgh Sect. A 126 (1996), 677-685.

[4] M. Wiegner, Decay results for weak solutions of the Navier-Stokes equations on $\mathbb{R}^{n}$, J. London Math. Soc. (2) 35 (1987), 303-313.

[5] M. Wiegner, Decay of the $L_{\infty}$-norm of solutions of the Navier-Stokes equations in unbounded domains, Acta Appl. Math. 37 (1994), 215-219.

[6] M. WIEgner, The Navier-Stokes equations - a neverending challenge?, Jahresber. Deutsch. Math.-Verein. 101 (1999), 1-25.

[7] M. Wiegner, Decay estimates for strong solutions of the Navier-Stokes equations in exterior domains, Ann. Univ. Ferrara Sez. VII (N.S.) 46 (2000), 61-79. 